\title{
Política de Saúde no Piauí: descentralização de recursos financeiros e resultados por estratos municipais
}

Jorge Otávio Maia BARRETO ${ }^{1}$

Inez Sampaio NERY ${ }^{2}$

\section{INTRODUÇÃO}

As políticas de saúde no Brasil se operacionalizam através do Sistema Único de Saúde (SUS), o qual foi concebido com escopo universal dentro do movimento denominado Reforma Sanitária e tem sua idealização estreitamente ligada à proclamação da saúde como direito de cidadania garantido pela Constituição Federal de $1988^{1}$. Os princípios informativos do SUS, sobre os quais toda a sua organização deve se fundamentar, são: a integralidade, universalidade e equidade no acesso à saúde ${ }^{1}$.

No entanto, tais princípios encontram limites à sua concretização pelo Estado, não querendo significar a garantia de acesso a tudo para todos, no âmbito da política de saúde. Antes disso, os diversos limites das políticas sociais universalistas, em especial no campo da saúde, exigem das sociedades estruturas normativas regulatórias complexas, que viabilizem sua operacionalização nos limites aceitos e suportados pela sociedade, tendo em conta que os custos dos sistemas de saúde universais, são, de forma geral, socializados através da arrecadação fiscal. Assim, o SUS consistiu, ao longo de mais de duas décadas de existência, um amplo e complexo repositório normativo, especialmente voltado para a dimensão organizacional do sistema, o qual está em constante evolução, exigindo constantemente a edição de normas operacionais específicas, em especial no âmbito da atribuição normativa da autoridade sanitária federal.

Uma das principais normas que tratam do papel do Estado no âmbito da saúde é a Lei Federal 8.080, de 1990, também denominada de Lei Orgânica da Saúde, que reafirma os princípios constitucionais do SUS e declara a saúde como direito humano fundamental a ser preservado pelo Estado brasileiro, a quem caberia prover as condições para seu exercícioº ${ }^{2}$ A Lei Orgânica da Saúde

1 Bacharelado em Direito pela Universidade Federal do Ceará (1995), especialista em Direito Processual (UESPI/2005), em Gestão da Saúde (ENSP/Fiocruz/2009) e em Economia e Avaliação de Tecnologias em Saúde (FIPE/ USP/2012). É mestre (UFPI/2010) e doutorando em Políticas Públicas (UFPI). Exerce o magistério superior desde 2001 e atualmente coordena o Curso de Bacharelado em Direito da Christus Faculdade do Piauí - CHRISFAPI, em Piripiri, Piauí.

2 Possui Graduação em Enfermagem pela Universidade Federal do Maranhão (1971), Mestrado em Enfermagem pela Universidade Federal do Rio de Janeiro/UFRJ/Escola de Enfermagem Ana Nery/EEAN (1980), Doutorado em Enfermagem pela UFRJ/EEAN (2000), Livre Docente pela Universidade do Rio de Janeiro/UNIRIO em 1991. Especialista em Metodologia do Ensino da Assistência de Enfermagem, Terapia Naturista, Produtos Naturais, Administração Hospitalar, Formação em Acupuntura pelo Centro de Acupuntura e Terapias Alternativas/CEATA-SP. 
também estabelece que o SUS é integrado pela União, estados e municípios, que devem desempenhar ações e serviços de saúde de forma complementar e participar da gestão e do financiamento do sistema de forma tripartite. Entretanto, essa mesma legislação é enfática na priorização do processo de descentralização de ações e serviços de saúde para os municípios, com o objetivo de edificar redes regionais e hierarquizadas em todos os níveis de assistência, com alocação racional de recursos financeiros e tecnológicos, a fim de buscar resultados e custos ótimos. Para tanto, cada município deveria assumir gradativamente as funções de 'gestor único' do sistema local, até atingir a plenitude da gestão em seu próprio território, inclusive financeira, executando de forma integral a política de saúde.

Os recursos que suportam o SUS são socializados, dado que provêm de toda sociedade e são captados pelo Estado através da arrecadação de tributos diretos e das contribuições da seguridade social. Estados e municípios estão obrigados pela Constituição Federal de 1988 a aplicar percentuais mínimos da sua arrecadação própria em ações de saúde, os quais correspondem respectivamente a 12 e $15 \%$, e a União deve destinar anualmente valor equivalente ao orçamento do ano anterior acrescido da variação do Produto Interno Bruto (PIB) daquele período. Os recursos sobre os quais a esfera federal dispõe, no entanto, representam a maior parcela de financiamento do SUS, tanto mais importante se tornando, quanto menor a capacidade de arrecadação própria dos estados e municípios.

O financiamento federal do SUS aporta nos Estados e Municípios através dos fundos de saúde, tal como previsto na Lei Federal 8.142, também de $1990^{3}$, que trata da participação social e das transferências intergovernamentais do sistema público da saúde. A lei 8.142 prevê a existência de recursos com parâmetro per capita destinados a financiar a assistência à saúde, os quais seriam transferidos 'fundo-a-fundo' pela União, de forma regular e automática, para os estados e municípios, conforme o respetivo estágio da gestão do SUS quanto à descentralização das ações de saúde nos seus níveis de complexidade, que vão da atenção primária à saúde (APS), promoção e vigilância em saúde, até a alta complexidade, que exige maior capacidade de investimento e envolve tecnologias de saúde cujo custeio é diferenciado e geralmente mais elevado. A APS se caracteriza como o primeiro nível de assistência, que abrange o cuidado longitudinal e integral, sendo apontada como a estratégia mais efetiva para a universalização do acesso à saúde ${ }^{4}$. De forma geral, as ações de APS deveriam ser desenvolvidas integralmente pelos municípios, cabendo-lhes a atribuição de organizar os sistemas locais para possuírem, no mínimo, assistência à saúde nesse nível.

Além das transferências 'não voluntárias' que a União está obrigada a realizar através do Fundo Nacional de Saúde para os fundos estaduais e municipais, também existem as transferências financeiras sob a forma de vários tipos de incentivos para investimento e custeio, os quais são condicionados à implantação de ações específicas, especialmente por adesão a políticas ou programas // Tempus, actas de saúde colet, Brasília, 8(3), 157-167, set, 2014. 
federais, visando, quase sempre, à implantação de novos serviços de saúde ou reorganização daqueles já existentes, para adequação ao preconizado pela instância federal do SUS, no exercício da sua atribuição normativa.

Nesse contexto, os municípios localizados nos estados brasileiros com menor capacidade de arrecadação têm buscado o suporte federal como principal fonte de financiamento das ações de saúde, em especial na APS, a qual todos os municípios brasileiros estão vinculados como atribuição inexcusável da administração local. O modelo de APS priorizado pelo SUS é a Estratégia Saúde da Família, consagrada na Política Nacional de Atenção Básica (PNAB) ${ }^{5}$ após mais de uma década da criação do Programa Saúde da Família ${ }^{6}$. A Saúde da Família consiste modelo assistencial de base comunitária, operacionalizado por equipes de saúde multidisciplinares, as quais são responsáveis pela população adstrita a um determinado território dentro do município, mediante o acompanhamento assistencial longitudinal das famílias e indivíduos, bem como da promoção de ações visando à prevenção e controle de riscos à saúde em nível coletivo.

As ações de APS, portanto, devem ser realizadas pelos municípios, sendo financiadas por recursos federais per capita fixos e por incentivos especiais variáveis, vinculados à implantação e manutenção de programas e políticas específicas criadas pela autoridade federal. A tais recursos federais se somam aqueles oriundos da arrecadação local, de competência tributária municipal, nas mesmas parcelas de aplicação obrigatória nas ações de saúde, além das 'eventuais' participações estaduais no financiamento direto da saúde através de transferências 'fundo-a-fundo'.

O gasto per capita anual em saúde dos municípios representa o conjunto destes recursos aplicados em razão do número de habitantes de um determinado território. O crescimento do gasto em saúde exige a ampliação da disponibilidade de recursos financeiros do SUS no território e certamente as transferências federais do SUS podem elevar o gasto em saúde dos municípios. Por outro lado, a elevação do gasto em saúde e da disponibilidade de recursos financeiros federais deveria significar o aumento do acesso aos serviços de saúde, o que, por sua vez, teria como resultado esperado a observação da melhoria nas condições de saúde da população, especialmente reduzindo adoecimentos e óbitos por causas consideradas evitáveis mediante o acesso aos serviços de saúde.

A situação de saúde de uma população pode ser monitorada mediante o uso de indicadores de saúde, geralmente relacionados ao perfil do adoecimento e do óbito em um território. A morbidade hospitalar reflete diretamente as condições gerais de vida de uma população, mas também é uma expressão dos níveis de acesso a serviços de saúde e da sua qualidade. No âmbito da APS, o cuidado em nível ambulatorial, seja preventivo ou terapêutico, pode evitar o aparecimento e o agravamento de doenças e, por conseguinte, a ocorrência da internação hospitalar por causas sensíveis a esse nível de assistência. 
Várias são as investigações que abordaram a relação entre as internações hospitalares e a atenção ambulatorial, a fim configurar indicador de qualidade e acesso aos serviços de saúde a partir do perfil das hospitalizações ${ }^{7,8,9,10,11}$. O Brasil já conta com uma lista oficial de Internações por Condições Sensíveis à Atenção Primária (ICSAP), lançada em 2008 pela Secretaria de Atenção à Saúde do Ministério da Saúde ${ }^{12}$. A lista contém dezenove grupos de causas de internação hospitalar, segundo a Décima Revisão da Classificação Internacional de Doenças (CID-10).

Considerando que as internações por causas sensíveis à presença de serviços de APS representam um indicador de acesso e qualidade, pode-se pressupor que a ampliação da disponibilidade de recursos para a implantação de serviços de saúde deveria alterar o perfil e a frequência da morbidade hospitalar passível de ser por ela afetada, significando a melhoria geral da situação de saúde.

O Estado do Piauí, localizado na Região Nordeste do país, apresenta a maior cobertura estimada de APS no Brasil, pelos parâmetros adotados pelo Ministério da Saúde, dado que a Estratégia Saúde da Família está presente em todos os municípios piauienses e 98,2\% da população estariam cobertos pelos serviços de APS no estado ${ }^{13}$.

A expansão dos serviços de APS observada no Piaú se deu ao longo da década de 2000, quando os municípios piauienses aderiram massivamente à política, deslanchando o processo de implantação da Saúde da Família no estado, com a consequente captação de recursos financeiros adicionais ao Piso da Atenção Básica (PAB) per capita, em face dos incentivos de investimento e custeio decorrentes da implantação dessas equipes multidisciplinares, compostas por médicos, enfermeiros, dentistas, técnicos auxiliares e agentes comunitários de saúde.

Entretanto, são pouco conhecidos os impactos produzidos pela Saúde da Família no Piauí em suas diversas dimensões, especialmente no que diz respeito à mudança do perfil da internação hospitalar no estado, embora BARRETO e colegas ${ }^{14}$ já tenham identificado, em estudo com o objetivo de analisar as mudanças no perfil de morbidade hospitalar entre crianças no Piauí, que apesar da redução das internações hospitalares observadas no período de 2000 a 2010, a proporção de ICSAP se manteve ou aumentou na faixa etária ate 5 anos, em especial por gastrenterites infecciosas, infecções respiratórias e asma.

Este estudo objetivou analisar o desempenho geral da APS no sistema público de saúde nos municípios do Piauí, agregados segundo o porte populacional, mediante a análise da correlação entre as transferências federais do SUS e as internações hospitalares sensíveis aos serviços de APS, no período de 2003 a 2010.

\section{MÉTODOS}

A fim de identificar aspectos gerais da relação entre as transferências federais do SUS e o // Tempus, actas de saúde colet, Brasília, 8(3), 157-167, set, 2014.

ISSN 1982-8829 
desempenho da política de saúde no estado, no período de 2003 a 2010, se analisou o comportamento de duas variáveis especialmente relevantes para medir o desempenho geral dos sistemas de saúde, considerando o ingresso de recursos e a produção de resultados: i) valores anuais de transferências federais do SUS per capita e ii) taxas de ICSAP, por 10 mil habitantes, na população geral. Pressupondo homogeneidade socioeconômica estre os municípios piauienses segundo perfis populacionais, esta análise agregou os resultados municipais nos seguintes estratos: i) até 20 mil habitantes; ii) 20-50 mil habitantes; iii) 50-100 mil habitantes e iv) mais de 100 mil habitantes. Os valores per capita e as taxas de ICSAP foram calculadas para os estratos populacionais considerando-se as populações e valores globais dentro de cada estrato.

Os dados foram coletados junto às bases oficiais disponíveis do Sistema de Informação sobre Orçamentos Públicos em Saúde (SIOPS) e do Sistema de Informações Hospitalares (SIH), ambos sob a responsabilidade do Ministério da Saúde, através do seu Departamento de Informática do SUS (DATASUS). Os dados populacionais foram obtidos nas bases do Instituto Brasileiro de Geografia e Estatística (IBGE) ${ }^{15}$. Após a coleta, organização e agregação dos municípios segundo estratos de porte populacional escolhidos, as séries temporais dos dois indicadores foram testadas quanto à correlação entre si em cada agregado de municípios, utilizando-se o coeficiente de correlação de Pearson, que retorna a estatística de correlação linear entre séries distintas com igual número de observações. Os resultados estão dispostos em gráficos e tabelas.

\section{RESULTADOS}

Os resultados apresentados na Figura 1 mostram que houve incremento relevante das transferências federais do SUS no período de 2003-2010 nos grupos de municípios analisados. As transferências federais do SUS para o Piauí superaram as médias nacional e regional em 2004 e mantiveram-se em proporções mais favoráveis ao estado até 2010 , quando alcançaram 18,1\% a mais que a média das transferências per capita do Nordeste.

No entanto, o aumento se deu de forma desigual nos diferentes estratos municipais. Observou-se que, apesar do estrato de municípios com população de 50-100 habitantes ter correspondido a $6,2 \%$ da população do estado em 2010, o incremento das transferências federais do SUS per capita chegou a 601,8\%, se comparados os anos de início e final da série histórica. O segundo maior incremento do valor médio per capita das transferências federais o SUS se deu no estrato dos municípios com menos de 10 mil habitantes, 190,5\%, bem inferior, portanto, ao aumento observado estrato mais beneficiado no período. Em seguida vêm os municípios com 2050 mil habitantes $(183,8 \%)$ e os maiores, com mais de 100 mil habitantes, que no Piaú são apenas dois, Parnaíba e a capital, Teresina, que receberam 122,8\% mais recursos federais do SUS, quando comparados os anos de 2003 e 2010. 
Figura 1 - Evolução do valor per capita das transferências federais do SUS dos agregados de municípios segundo porte populacional, Piaui, 2003-2010.

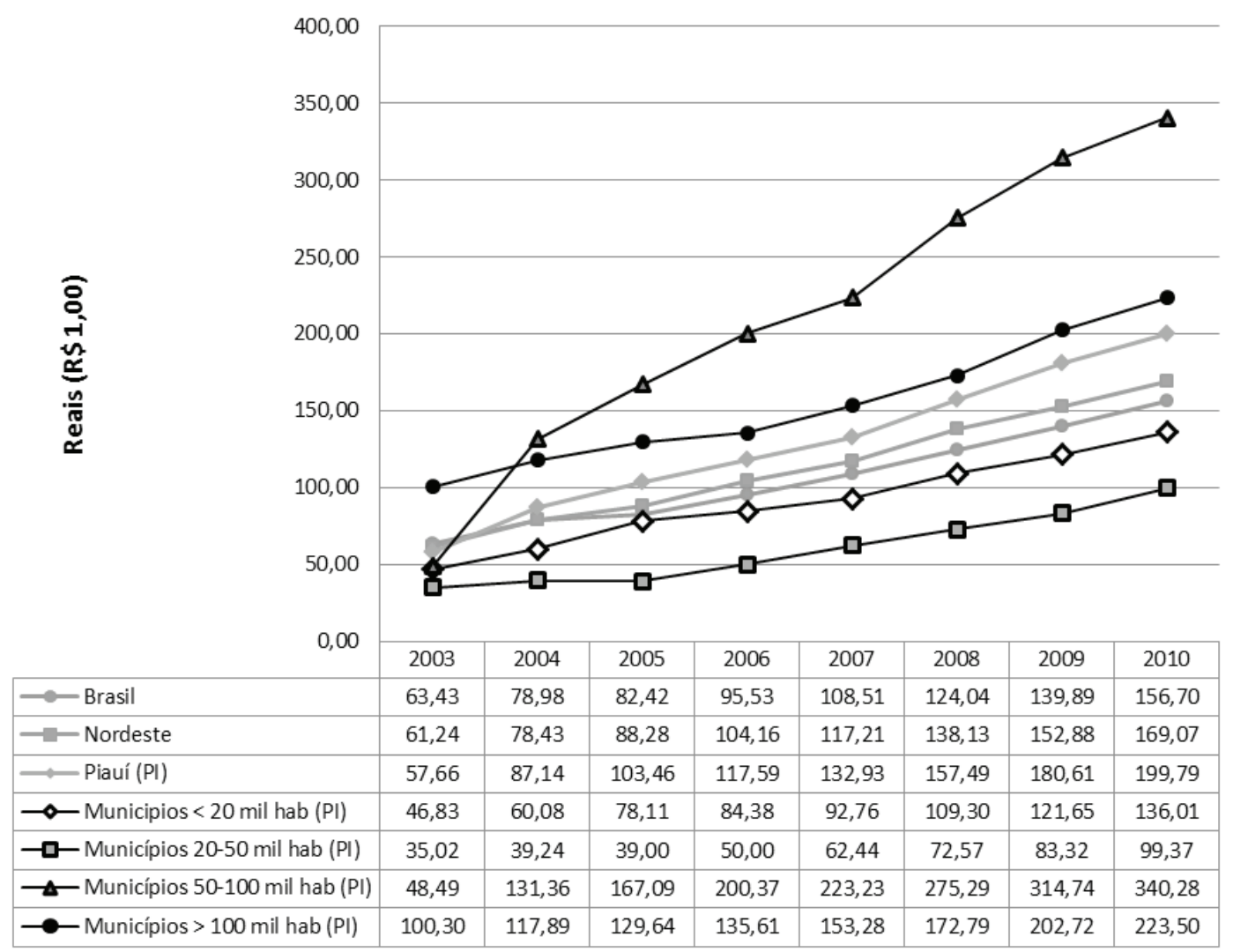

Fonte: SIOPS/DATASUS/Ministério da Saúde (dados sobre orçamentos públicos); IBGE (dados populacionais).

Percebeu-se que municípios maiores têm recebido mais recursos federais, considerando-se o per capita total, especialmente aqueles com 50-100 mil habitantes, que apresentaram, ao longo da série analisada, os maiores valores, seguidos dos municípios com mais de 100 mil habitantes, exceto no ano de 2003, quando as posições entre os maiores municípios apareciam invertidas.

Um aspecto relevante a ser incluído nesta análise é a proporção da população piauiense que se insere em cada estrato populacional. Segundo os dados do Censo Demográfico de 2010 do IBGE, os municípios com menos de 20 mil habitantes abrigavam $43 \%$ da população piauiense, os de 20-50 mil, 20\%, os com mais de 100 mil habitantes, 30,8\% e os com população de 50-100, como já dito, 6,2\%. Portanto, a evolução do valor per capita médio das transferências federais do SUS no Piauí parece beneficiar municípios maiores, em especial aqueles com população de 50-100 mil habitantes, que, embora correspondam à menor parcela da população, tanto receberam maior incremento como apresentaram valores superiores aos estratos dos municípios de menor população.

Tal pode se dever a vários fatores, mas é provável que se deva à concentração de serviços financeiramente melhor recompensados pelo SUS, em face da sua complexidade, tais como serviços hospitalares e a utilização nos municípios mais populosos das tecnologias em saúde indisponíveis nos municípios menores. Entretanto, essa análise não enfoca a explicação sobre 
estas divergências quanto às transferências federais do SUS para os municípios agregados por porte populacional, mas, na etapa seguinte, examinará como as internações hospitalares por causas potencialmente evitáveis pelo acesso oportuno a serviços de APS se comportaram no período de 2003 a 2010.

A Figura 2 apresenta a evolução das taxas de internação hospitalar, calculadas por 10 mil habitantes nos agregados de municípios por porte populacional, a partir dos dados do SIH e IBGE. Também mostra os resultados apresentados por Dourado e colaboradores (2011) em estudo para avaliar o impacto da implantação da estratégia Saúde da Família sobre as ICSAP em nível nacional, regional e estadual, de 1999 a 2007.

O que se verificou é que houve redução das ICSAP em todos os estratos municipais, exceto no estrato de municípios com população de 20-50 mil habitantes, que, ao contrário, aumentou sua taxa de ICSAP em 27,5\%. No outros estratos, as reduções variaram de magnitude, sendo pequenas no grupo dos municípios com até 20 mil habitantes $(7,7 \%)$ e ainda menores nos municípios com 50100 mil habitantes $(3,8 \%)$, e elevadas nos municípios de maior porte, os quais chegaram a reduzir em $32,5 \%$ as suas ICSAP, comparando-se os extremos da série.

Figura 2 - Evolução da Taxa de ICSAP dos agregados de municípios segundo porte populacional, Piaui, 2003-2010.

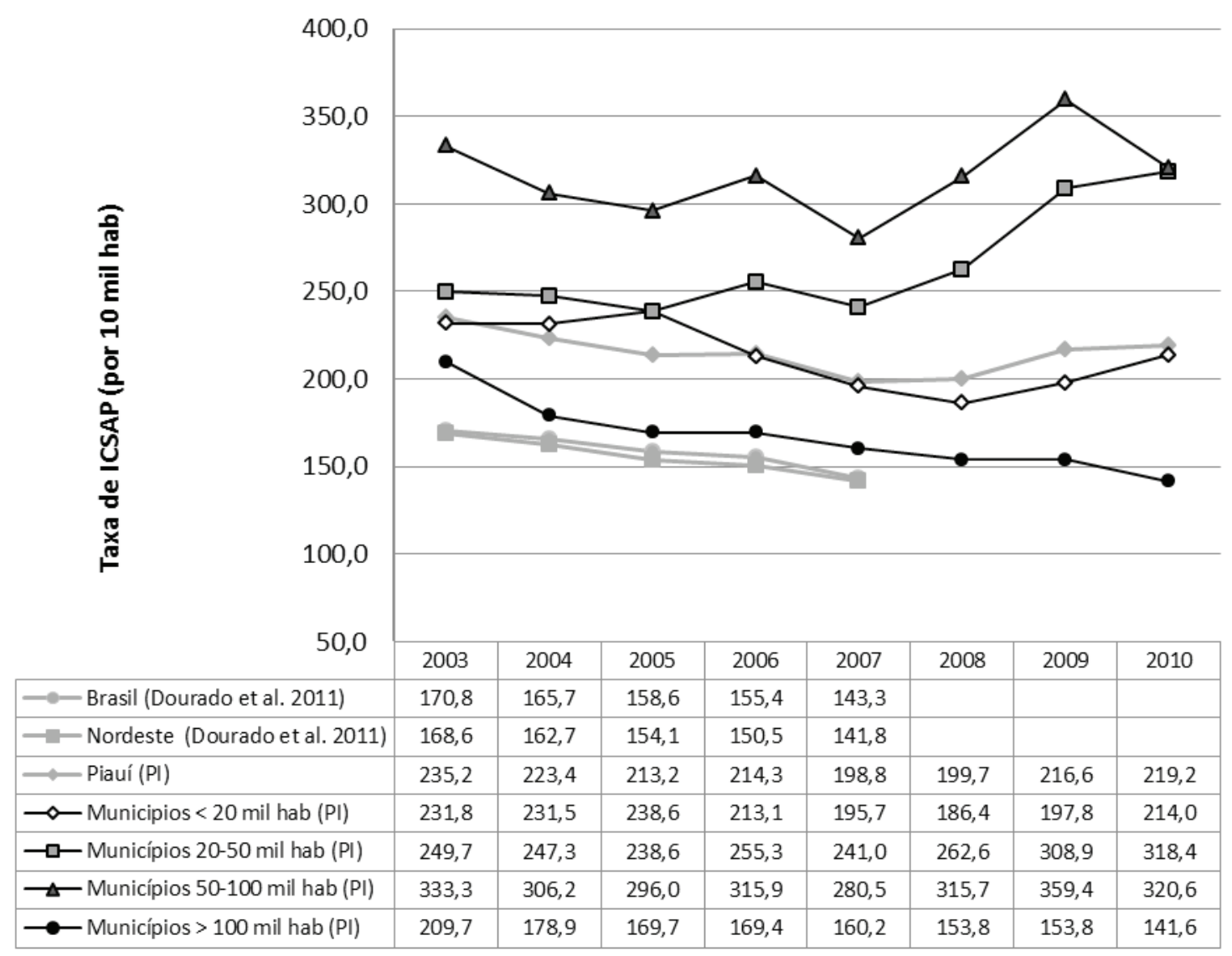

Fontes: Piauí, 2003-2010 - SIH/DATASUS/Ministério da Saúde (dados hospitalares) e IBGE (dados populacionais); 
Importante ressaltar que as taxas de ICSAP apresentadas ainda devem ser consideradas elevadas para todos os estratos, com exceção dos municípios com mais de 100 mil habitantes, se consideradas as médias nacionais. Conforme estudo recente de abrangência nacional sobre as taxas de internação hospitalar por condições sensíveis à atenção ambulatorial, a taxa média brasileira em 2007 estava no patamar de 143,3, enquanto o Nordeste apresentava 141,8, no mesmo ano ${ }^{16}$. Assim, em todos os anos da série analisada as taxas de ICSAP estiveram efetivamente muito superiores aos padrões registrados por Dourado e colaboradores ${ }^{16}$.

Entretanto, a pior situação está no estrato de municípios com 50-100 mil habitantes, onde foi verificado o aumento da taxa de ICSAP, sendo seguido pelo estrato de 20-50 mil e pelo estrato de município com até 20 mil habitantes, que registraram pequenas reduções. A melhor evolução foi observada no estrato dos municípios com população de 100 mil habitantes, com uma redução comparada entre 2003 e 2010 de 32,5\%. A taxa de ICSAP do Piauí, por sua vez variou negativamente em $6,8 \%$, entre 2003 e 2010.

Quando testada a correlação linear entre a evolução das transferências federais do SUS e das taxas de ICSAP por estrato populacional municipal (Tabela 1), verificou-se que o aumento das transferências federais do SUS para municípios até 20 mil habitantes apresentou forte correlação negativa com a redução das taxas de $\operatorname{ICSAP}(r=-0,65)$, ao passo que para os municípios de 20-50 mil habitantes a correlação obtida foi também foi forte, mas positiva, implicando que o aumento das transferências federais e das ICSAP andaram juntas no período analisado $(\mathrm{r}=0,87)$.

\begin{tabular}{lcccc}
\hline Municípios por estrato populacional & $\mathrm{N}$ & \% municípios & \% População & $\boldsymbol{r}$ \\
Até 20 mil habitantes & 199 & $88,8 \%$ & $43,0 \%$ & $-0,65$ \\
$20-50$ mil habitantes & 20 & $8,9 \%$ & $20,0 \%$ & 0,87 \\
$50-100$ mil habitantes & 3 & $1,3 \%$ & $6,2 \%$ & 0,21 \\
mais de 100 mil habitantes & 2 & $0,9 \%$ & $30,8 \%$ & $-0,88$ \\
\hline Total & $\mathbf{2 2 4}$ & $\mathbf{1 0 0 \%}$ & $\mathbf{1 0 0 \%}$ & \\
\hline
\end{tabular}

Fonte: IBGE (dados populacionais)

Para os municípios com 50-100 mil habitantes, apesar de apresentarem as maiores taxas de ICSAP e os maiores valores per capita de transferências federais do SUS, a correlação entre estas se mostrou fraca positiva $(\mathrm{r}=0,21)$, ao contrário do estrato dos maiores municípios, com mais de 100 mil habitantes, que mostraram forte correlação negativa $(r=-0,88)$ entre a evolução das transferências federais e as taxas de ICSAP no período analisado. 
A Tabela 1 também apresenta os dados da proporção que cada agregado de municípios representa no total da população do Estado do Piauí, quanto ao número de municípios naquela faixa de população e a população residente correspondente, segundo o Censo Demográfico de 2010.

\section{DISCUSSÃO E CONSIDERAÇÕES FINAIS}

A principal limitação desta análise foi o uso de dados municipais agregados em estratos segundo o porte populacional. Efetivamente tal não permitiu identificar situações municipais diferenciadas, pois a agregação das variáveis analisadas incorpora melhores e piores resultados em cada estrato populacional tratando-os como homogêneos dentre de cada grupo de municípios.

Entretanto, tal limitação não invalida o esforço para o reconhecimento das tendências gerais quanto às transferências federais do SUS e ICSAP entre municípios com populações diferentes no Piauí, dado que comparações entre grupos de municípios agregados por estratos populacionais pressupõem características assemelhadas neste aspecto entre os municípios que integram cada estrato. Variações no desempenho dos sistemas locais são plausíveis e podem ser objeto de novas investigações que abordem o município como unidade de análise.

Verificou-se que as transferências federais no âmbito das políticas de saúde operacionalizadas pelo SUS no Piauí, pelo menos no período de 2003 a 2010, sofreram incrementos muito relevantes, mas também desiguais quanto ao seu crescimento e valores absolutos, se considerados os valores médios dos municípios agregados por porte populacional.

Os mais beneficiados com transferências federais da saúde foram os de maior porte, que tiveram maiores aumentos e apresentam maiores valores de transferências per capita, em especial aqueles situados na faixa de 50-100 mil habitantes. Os municípios de menor porte também apresentaram aumento do recebimento de recursos federais no período, mas os valores per capita das transferências federais do SUS mostraram-se significativamente inferiores dos municípios de maior porte.

Por outro lado, a situação de saúde relacionada com as internações hospitalares por causas especialmente evitáveis pelo acesso oportuno a serviços ambulatoriais de qualidade, tais como os previstos pela Estratégia Saúde da Família, a qual tem cobertura estimada de 98,2\% da população piauiense, sofreu pouca mudança nos período analisado, especialmente naqueles municípios de menor porte, permanecendo em patamares pouco alterados ao longo dos oito anos analisados. Tal contrariou a expectativa de que a ampliação do acesso aos serviços de saúde poderia ter interferido na frequência e perfil das internações hospitalares no Piauí, especialmente naquelas relacionadas a causas sensíveis à APS.

É possível supor, a partir desta análise, que os melhores resultados relacionados com as ações de saúde no Piauí incentivadas pelas transferências federais do SUS ainda não foram alcançados nos municípios compreendidos nos grupos de 20 a 100 mil habitantes, onde as correlações verificadas entre a evolução das transferências federais e as ICSAP foram positivas, ou seja, o aumento das 
transferências foi acompanhado do aumento das internações hospitalares sensíveis à APS.

Já para municípios piauienses localizados nos estratos extremos, ou seja, com menos de 20 mil e com mais de 100 mil habitantes, os quais correspondem juntos a 73,8\% da população piauiense, as transferências do SUS e as taxas de ICSAP mostraram-se inversamente proporcionais.

Em 2010, ainda foram altas as taxas de ICSAP e baixos os valores per capita transferidos pela União através dos mecanismos de financiamento das políticas de saúde, seja por transferências obrigatórias ou por incentivos por ações específicas. Tal observação reforça a necessidade de novos estudos para identificação das reais causas das diferenças entre os grupos de municípios analisados, bem como do baixo desempenho quanto à redução das ICSAP na maior parte do estado.

\section{REFERÊNCIAS BIBLIOGRÁFICAS}

1. Brasil. Constituição. Constituição da República Federativa do Brasil (1988). Brasília: Senado Federal; 2006.

2. Brasil. Lei n. 8080, de 19 de setembro de 1990. Dispõe sobre as condições para a promoção, proteção e recuperação da saúde, a organização e o funcionamento dos serviços correspondentes. Diário Oficial da União, Brasília, v.78, n.182, p. 18055, 20 set. 1990. Seção 1.

3. Brasil. Lei n. 8142, de 28 de dezembro de 1990. Dispõe sobre a participação da comunidade na gestão do Sistema Único de Saúde - SUS e sobre as transferências intergovernamentais de recursos financeiros na área da saúde. Diário Oficial da União, Brasília, v.78, n.249, p.25694, 31 dez. 1990. Seção 1.

4. Organização Mundial de Saúde. Declaração de Alma-Ata. In: Conferência Internacional sobre Cuidados Primários de Saúde, Brasília: OMS; 1979.

5. Ministério da Saúde (Brasil). Política Nacional de Atenção Básica. Brasília: Ministério da Saúde; 2006.

6. Ministério da Saúde (Brasil). Saúde da Família: Uma Estratégia para a Reorientação do Modelo Assistencial. Brasília: Ministério da Saúde; 1998.

7. Márquez-Calderón $\mathrm{S}$ et al. Factores asociados a la hospitalización por procesos sensibles a cuidados ambulatorios en los municipios. Gac Sanit. 2003;17:360-7..

8. Malta DC, Duarte EC, Almeida MF. Lista de causas de mortes evitáveis por intervenções do Sistema Único de Saúde do Brasil. Epidemiol Serv Saúde. 2007; 16(4):233-44.

9. Fernandes VBL et al. Internações por condições sensíveis. Rev Saúde Pública. 2009;43(6):928- 
36.

10. Alfradique ME et al. Internações por condições sensíveis à atenção primária: a construção da lista brasileira como ferramenta para medir o desempenho do sistema de saúde (Projeto ICSAP - Brasil). Cad Saúde Pública. 2009; 25(6): 1337-49.

11. Moura BLA et al. Principais causas de internação por condições sensíveis à atenção primária no Brasil: uma análise por faixa etária e região. Rev Bras Saúde Matern Infant. 2010; 10(supl.1):s83-s91.

12. Ministério da Saúde (Brasil). Secretaria de Atenção à Saúde. Portaria 221, de 17 de abril de 2008. [Acesso em 20 de abril de 2012]. Disponível em: http://dtr2001.saude.gov.br/sas/PORTARIAS/ Port2008/PT-221.htm

13. Ministério da Saúde (Brasil) [homepage na internet]. Secretaria de Atenção à Saúde. Departamento de Atenção Básica. Página do Departamento de Atenção Básica. Brasília: Ministério da Saúde. [Acesso em 25 de abril de 2012]. Disponível em: http://dtr2004.saude.gov.br/dab/historico cobertura_sf.php

14. Barreto JOM, Nery IS, Costa MSC. Estratégia Saúde da Família e internações hospitalares em menores de 5 anos no Piauí, Brasil. Cad Saúde Pública 2012; 28(3):515-26.

15. Instituto Brasileiro de Geografia e Estatística - IBGE (Brasil). [homepage na internet]. Censos Demográficos. Sinopse do Censo Demográfico de 2010. [Acesso em 30 de abril de 2012]. Disponível em: http://www.ibge.gov.br/estadosat/temas.php?sigla=pi\&tema=sinopse_censodemog2010

16. Dourado I et al. Trends in Primary Health Care-sensitive Conditions in Brazil: the role of the Family Health Program (Project ICSAP-Brazil). Medical Care 2011; 49(6): 577-84.

Artigo apresentado em 21/02/14

Artigo aprovado em 12/08/14 Artigo publicado no sistema em 21/09/14 